\title{
Surface energetic effects of human immunodeficiency virus co-infection on mycobacterium tuberculosis-macrophage interaction mechanism
}

\begin{abstract}
The mechanism of mycobacterium tuberculosis macrophage interactions was studied using surface energetics tool in determining the interaction processes. The surface interfacial energies were explained using van der Waals concept of particle interactions. Twenty samples each of infected and uninfected sputum were collected and screened to determine the infection status using GeneXpert and Ziehl-Neelsen staining method. The absorbance, $\bar{a}$, values of each specimen, for wavelength range of $230-950 \mathrm{~nm}$ were measured using digital Ultraviolet Visible Spectrophotometer. Matlab software tools were used in the mathematical analysis of the data generated from the absorbance values. The combined Hamaker coefficients were obtained using the values of the dielectric constant with the Lifshitz Equation. The harmonized Hamaker coefficients $\mathrm{A}_{132 \mathrm{har}}$ and the absolute combined Hamaker coefficients $\mathrm{A}_{132 \mathrm{abs}}$ of infected samples were deduced. The values of $\mathrm{A}_{132 a b s}$ for $m t b$ infected sputum and $\tilde{\mathrm{A}}_{132 \mathrm{abs}}$ for $m t b / \mathrm{HIV}$ co-infected sputum are $0.21631 \times 10^{-21} \mathrm{Joule}$ and $0.18825 \times 10^{-21} \mathrm{Joule}$ respectively. This result reveals that the surface energy of the macrophage is less than the surface energy of the $m t b, m t b / \mathrm{HIV}$ while the surface energy of the $m t b / \mathrm{HIV}$ macrophage is less than that of $m t b$ macrophage. HIV has the tendency to reduce the interaction energy with the consequences of increased viral loads and decreased immune systems. The interaction energy is reduced by $13 \%$ confirming adverse effects observed in HIV patients suffering from $\mathrm{Tb}$. Negative Hamaker coefficient of $-0.22669 \times 10^{-19} \mathrm{~mJ} / \mathrm{m}^{2}$ was calculated indicating that separation of $m t b$ is practical. The effect of the infection can only be abated if additive(s) in form drug is added that can render $\mathrm{A}_{132}$ negative. Thus, the value of Hamaker constant of the sputum $\mathrm{A}_{33}$ was required for repulsion to occur mathematically derived as $\mathrm{A}_{33} \geq 0.9527 \times 10^{-21}$ Joule which satisfies this condition for negative $\mathrm{A}_{132 a b s}$
\end{abstract}

Keywords: absorbance, energetics, hamaker coefficient, macrophage, mycobacterium tuberculosis, van der waals forces, wavelength
Volume 3 Issue 6 - 2017

\author{
Chukwuneke L Jeremiah,' Achebe $\mathrm{H}$ \\ Chinonso,' Sinebe E Jude, ${ }^{2}$ Ugwuegbu C \\ Duke $^{3}$ \\ 'Department of Mechanical Engineering, Nnamdi Azikiwe \\ University, Nigeria \\ ${ }^{2}$ Department of Mechanical Engineering, Delta State University, \\ Nigeria \\ ${ }^{3}$ Department of Mechanical Engineering, Federal Polytechnic, \\ Nigeria
}

\author{
Correspondence: Chukwuneke L Jeremiah, Department \\ of Mechanical Engineering, Nnamdi Azikiwe University, Awka, \\ Nigeria, Tel 234(0)8033574353, \\ Email jl.chukwuneke@unizik.edu.ng
}

Received: November II, 2016 | Published: September 21, 2017

\section{Introduction}

In the world today, tuberculosis $(t b)$ is the leading cause of death due to its interaction with other infectious diseases such as the Human Immunodeficiency Virus (HIV), which is the AIDS causative agent. ${ }^{1}$ The HIV impact on tb is quite overwhelming prior to the fact that tb would already be declining worldwide if not for HIV which is contributing approximately $10 \%$ of all global mortality cases. ${ }^{2}$ Individual who are latently infected have a $10 \%$ risk of developing the disease in their life time, although HIV is a strong risk factor for developing active tb. ${ }^{3-5}$ About 14 million people are estimated worldwide with active tb infection. However, the vast majority of tbrelated deaths occur in the developing part of the world. ${ }^{6}$ About 9.4 million new tb cases were reported in 2009, and about $22.5 \%$ of 1.7 million deaths reported were related to tb among people with HIV. ${ }^{6}$ About 8.7 million people suffered from tb-related illness, including 1.1 million cases among people with HIV, leading to 1.4 million deaths in 2011. ${ }^{7}$ The bacterial pathogen that causes tb mycobacterium tuberculosis $(m t b)$ latently infects one third of the world's population and is responsible for the highest mortality rate of any single bacterial pathogen., ${ }^{3,4}$ The lung is typically the primary site of the bacterial infection, where it is engulfed by macrophages during an innate immune response. ${ }^{1}$ Mycobacterium tb has a slow doubling time of about 15-20 hours, and prefers to live within macrophages. Bacteria are then transported by dendritic cells to the closest draining lymph nodes (LN) where the host's adaptive immune response is mounted against infection.

A T-cell mediated immune response rather than an antibody mediated immune response is necessary to combat infection since $m t b$ generally resides within macrophages. These take up the bacteria together with the dendritic cells at the site of infection and transport them or bacterial antigen to the draining $\mathrm{LN}$ where a specific immune

response can be developed. $C D_{4}^{+}$T-cells and macrophages are critical to our immune system response and HIV is a virus that specially infects them, ${ }^{1}$ thus compromising the host ability to control interaction(s). HIV weakens the immune system; $t b$ thrives in a weakened immune system. Each disease increases the other's progress. ${ }^{8,9}$ Surface property determination of interacting particles lead to the further understanding of the mechanism of interactions; whereby a common area of contact is established once two particles meet each other leading to a displacement of a certain portion of each particle. The 
consecutive impact on the surface is called surface thermodynamic effects. From surface thermodynamic point of view, related works are in the phagocytic engulfment and cell adhesiveness as cellular surface phenomena and bacteria platelets highly developed by. Van Oss et al., ${ }^{10}$ Stuart, ${ }^{11}$ Absolom et al., ${ }^{12}$ Neumann et al. ${ }^{10}$ considered the relationship between free energy of engulfment and number of bacteria ingested. In this research, it is of interest to understand how the $m t b$ interacts with the macrophage. The process involves the bacterium modeled as a particle in a liquid medium (sputum) attaching itself on the surface of the macrophage (another particle), penetrating and probably destroying it. The condition under which bacterium attachment on the surface of the macrophage does not take place will be sought, even in the presence of HIV with its destructive effect. The free energy for the condition where no adhesion should occur is to be predicted from the concept of negative Hamaker coefficient. It is also desired to determine quantitatively the mechanism of interactions between the $m t b$ and macrophages using the concept of surface energetics.

\section{Methodology}

The methodology of this research work involved sputum sample collection, mycobacterium and macrophages structural studies, mycobacterium tuberculosis screening, and the study of the mechanism of interaction of the bacterium and the macrophage. Twenty samples each of infected, uninfected and $m t b / \mathrm{HIV}$ co-infected sputum was collected. Each specimen was screened to determine the infection status using GeneXpert and Ziehl-Neelsen staining method. The slides were prepared and the absorbance, $\bar{a}$, values of each specimen, for wavelength range of $230-950 \mathrm{~nm}$ were measured using digital Ultraviolet Visible Spectrophotometer. The data generated and the various Equation s governing the relationship among the variables were used in calculating values for the reflectance, $\mathrm{R}$, transmittance, $\mathrm{T}$, refractive index, $\mathrm{n}$, and the dielectric constant, $\varepsilon$. Matlab software tools were employed in the mathematical analysis of the data generated from the absorbance values.

\section{Thermodynamics particle-particle interaction me- chanisms}

Presume in a certain case, the bacterium, $m t b$ has been considered as a particle coming in the vicinity of the macrophage which is further considered as another particle. In the successive step, one particle (the bacterium) attaches itself on the surface of the other (the macrophage) under a specific condition where the macrophage is dispersed in sputum medium (Figure 1). Expression of the thermodynamic free energy associated with adhesion process $\Delta F_{p l s}^{a d h}$ provided in Figure 1 is represented as follows: ${ }^{13,14}$

$$
\Delta F_{p l s}^{a d h}\left(d_{o}\right)=\gamma_{p s}-\gamma_{p l}-\gamma_{s l}
$$

Where,

$\Delta F_{p l s}^{a d h}=$ The integrated free energy of adhesion considered from infinity to the equilibrium

$\mathrm{d}_{\mathrm{o}}=$ separation distance

$P \& S=$ Bacteria and macrophage respectively

$\mathrm{L}=$ sputum

$\gamma_{\mathrm{ps}}=$ Expresses the interfacial free energy between $\mathrm{P}$ and $\mathrm{S}$ $\gamma_{\mathrm{pl}}=$ Expresses the interfacial free energy between $\mathrm{P}$ and $\mathrm{L}$

$\gamma_{\mathrm{sl}}=$ Expresses the interfacial free energy between $\mathrm{S}$ and $\mathrm{L}$

Successful bacterial penetration in the macrophage cells will occur through the engulfment of the bacteria requiring net free energy, represented as: $:^{13,15}$

$$
\Delta F_{N E T}=\gamma_{p s}-\tilde{\mathrm{a}}_{\mathrm{pl}}<0
$$

In Equation 2, rejection of the bacteria by the macrophage will be represented, if $\Delta F_{N E T}$ becomes greater than zero. For the interaction between the individual components, similar Equation s can be written also: ${ }^{13,14}$

$$
\begin{aligned}
& \Delta F_{p s}^{a d h}\left(d_{1}\right)=\gamma_{p s}-\gamma_{p v}-\gamma_{s v} \\
& \Delta F_{s l}^{a d h}\left(d_{1}\right)=\gamma_{s l}-\gamma_{s v}-\gamma_{l v} \\
& \Delta F_{p l}^{a d h}\left(d_{1}\right)=\gamma_{p l}-\gamma_{p v}-\gamma_{l v}
\end{aligned}
$$

For a liquid, the force of cohesion, which is the interaction with itself, is described by:

$$
\Delta F_{i i}^{c o h}\left(d_{1}\right)=-2 \gamma_{l v}
$$

$\Delta \mathrm{F}^{\text {adh }}$ can be determined by several approaches, apart from the above surface free energy approach. The classical work of ${ }^{16}$ is very appropriate.

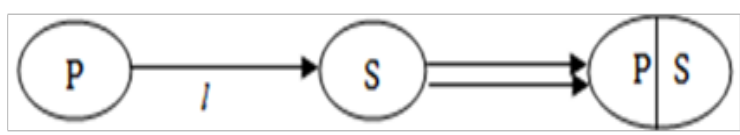

Figure I Conceptual representation of $m t b$-macrophage adhesion process.

A system containing two planes could be considered for computing the free energy of interaction. This can be done for semi-infinite, parallel bodies belonging to material 1 and 2 isolated by material 3 , bearing thickness 'd' (Figure 2) provided in the following section. ${ }^{16}$ This is calculated by the following Equation:

$$
\Delta F_{132}\left(d_{o}\right)=\left[-\frac{A_{132}}{12 \pi d_{o}^{2}}\right]
$$

In this, $A_{132}=$ Hamaker coefficient for a respective system.

Considering nominal isolation distance $\mathrm{d}_{0}$, and Equation 7 as valid for such a small distance, the Hamaker coefficient should be expressed as: $:^{16}$

$$
A_{132}=-12 \pi d_{o}^{2} \Delta F^{a d h}\left(d_{o}\right)
$$

The Hamaker coefficient $\mathrm{A}_{132}$ for the interactions between two different bodies in a liquid can be calculated from Equation 8 once the free energy of adhesion between the two bodies is known or through the pair-wise additivity approach as originally proposed by Hamaker ${ }^{16}$ or by the macroscopic approach of Lifshitz. ${ }^{17}$ As the actual material atomic structures are overlooked, the Lifshitz method is suitable in certain cases. In this method, bulk material properties are considered for calculation of interactions between the macroscopic bodies. 
Properties like refractive indices and dielectric permittivity $\varepsilon(i \zeta)$ are considered for such calculations. Dielectric permittivity represents the microscopic polarizability as a manifested macroscopic property for the constant atoms belonging to certain materials. The Hamaker coefficient represents the macroscopic resultant for the interactions which happened due to the atom polarizations in a material. ${ }^{18}$ Following Lifshitz theory, the Hamaker coefficient is represented as follows: ${ }^{17}$

$$
A_{i k j}=\frac{3}{4} \pi \hbar \int_{0}^{\infty}\left[\frac{\varepsilon_{i}(i \zeta)-\varepsilon_{k}(i \zeta)}{\varepsilon_{i}(i \zeta)+\varepsilon_{k}(i \zeta)}\right]\left[\frac{\varepsilon_{j}(i \zeta)-\varepsilon_{k}(i \zeta)}{\varepsilon_{j}(i \zeta)+\varepsilon_{k}(i \zeta)}\right] d \zeta
$$

Where, $\varepsilon_{j}(i \zeta)$ refers to the dielectric constant of a specific material $\mathrm{j}$, this is considered through the imaginary $\mathrm{i}$, frequency axis, $(i \zeta)$, $\square$ is planck's constant. In this context, the evaluation of Equation 9 should result in equivalent value with the thermodynamic free energy of adhesion, provided in Equation 1. The molecular contact was maintained at $(\mathrm{d}=0)$. Interestingly, constituent molecule numbers are of finite size and for that it is not possible to attain $d=0$ for two macroscopic surfaces. Therefore, whenever the surfaces attain a distance $d_{o}$, molecular contacts are considered. The divergences according to Lifshitz theory are eliminated by the parameter $\mathrm{d}_{\mathrm{o}}$. Thus, the Hamaker coefficient or the Lifshitz-van der Waals constant $\mathrm{A}_{132}$ may result in negative. In certain condition, the contact between the interacting particles will be hampered due to the repulsive (electrostatic) force originated. The resultant effect in certain cases remains as repulsion instead of attraction for the considered particles. The Hamaker coefficient and the interfacial free energies are connected through the following Equation:

$$
A_{p l s}=-12 \pi d_{o}^{2}\left(\gamma_{p s}-\gamma_{p l}-\gamma_{s l}\right)
$$

This Equation has been derived through combining Equation 8 with Equation 1. For the issue of self-interaction of a particle Equation 11 should be considered:

$$
A_{i j}=\frac{3}{4} \pi \hbar \int_{0}^{\infty}\left[\frac{\varepsilon_{i}(i \zeta)-\varepsilon_{j}(i \zeta)}{\varepsilon_{i}(i \zeta)+\varepsilon_{j}(i \zeta)}\right]^{2} d \zeta
$$

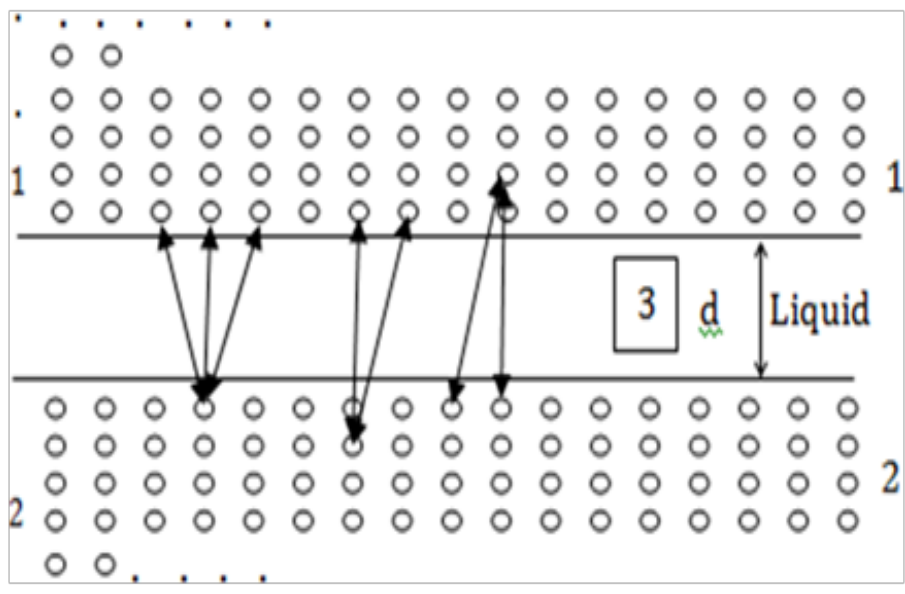

Figure 2 Representation of interaction of two solid bodies, depicted by I and 2 which are eventually isolated by d, liquid 3 .
To be able to use the absorbance data to calculate the Hamaker coefficients using the Lifshitz theorem of Equation 9, there is a need to evaluate the dielectric constant $\varepsilon$ of the Equation. From the information of light absorbance, reflection and transmittance, it could be seen that:

$$
\hat{a}+T+R=1
$$

Where; $\bar{a}$ is absorbance, $\mathrm{T}$ is transmittance, and $\mathrm{R}$ is reflectance. Also, from the information of light absorbance and transmittance:

$$
T=\exp ^{-\hat{a}}
$$

With the values of $\bar{a}$ determined from absorbance experimental results and substituting the values of $\bar{a}$ into Equation 13 to obtain $T$, $R$ could easily be derived by substituting the values of $\bar{a}$ and $T$ into Equation 12. To find a value for the refractive index, $n$ employing the mathematical relation: ${ }^{19}$

$$
n=\left[\frac{1-R^{1 / 2}}{1+R^{1 / 2}}\right]
$$

A value for the extinction coefficient, $k$ is obtained from the Equation:

$$
k=\left[\frac{\alpha \lambda \times 10^{-9}}{4 \pi}\right]
$$

Where; $\alpha$ is the absorption coefficient defined as follows:

$$
\alpha=\left[\frac{\bar{a}}{\lambda \times 10^{-9}}\right]
$$

Substituting the value, $\alpha$ of Equation 16 into Equation 15:

$$
k=\left[\frac{\bar{a}}{4 \pi}\right]
$$

The dielectric constant, $\varepsilon$ could thus be given by the formula, ${ }^{20}$ for the real part:

$$
\varepsilon_{1}=n_{1}^{2}-k^{2}
$$

For the imaginary part:

$\varepsilon_{2}=2 n_{2} k$

With these values, it is possible to determine $A_{i j}$ using the relevant Equation $s$ to determine $\mathrm{A}_{11}$ :

$$
A_{11}=2.5\left[\frac{\varepsilon_{10}-1}{\varepsilon_{10}+1}\right]^{2}=2.5\left[\frac{n_{1}^{2}-1}{n_{1}^{2}+1}\right]^{2}
$$

This gives a value to the Hamaker constant $\mathrm{A}_{11}$ and by extension to other Hamaker constants $\mathrm{A}_{22}$ and $\mathrm{A}_{33}$. For combination of two different materials 1 and 2 in approximation:

$$
A_{12}=\sqrt{A_{11} A_{22}}
$$


For a combination of two dissimilar materials (i.e. macrophage, 1 and the bacteria, 2) with the gap between 1 and 2 filled with sputum as the medium 3, the combined Hamaker coefficient will be given by:

$$
\begin{aligned}
& A_{132}=A_{12}+A_{33}-A_{13}-A_{23} \\
& A_{131}=A_{11}+A_{33}-2 A_{13}
\end{aligned}
$$

Therefore, the system under consideration follows Equations 22 an 23. Rewriting these Equations will give:

$$
\begin{aligned}
& A_{132}=\left(\sqrt{A_{11}}-\sqrt{A_{33}}\right)\left(\sqrt{A_{22}}-\sqrt{A_{33}}\right) \\
& A_{131}=\left(\sqrt{A_{11}}-\sqrt{A_{33}}\right)^{2}
\end{aligned}
$$

Equation 24 shows that, for a three-component system involving three different materials, 1, 2 and 3, $\mathrm{A}_{132}$ can become negative:

$$
A_{132}<0
$$

When,

$$
\sqrt{A_{11}}>\sqrt{A_{33}} \text { and } \sqrt{A_{22}}<\sqrt{A_{33}}
$$

$\mathrm{A}_{33}=$ Hamaker constant for sputum; $\mathrm{A}_{13}=$ Hamaker constant between both materials (i.e. $m t b$ and sputum); $\mathrm{A}_{23}=$ Hamaker constant between both materials (i.e. macrophage and sputum). Thus the Hamaker Coefficient $\mathrm{A}_{132}$ becomes:

$$
A_{132}=\frac{3}{4} \pi \hbar \int_{0}^{\infty}\left[\frac{\varepsilon_{1}(i \zeta)-\varepsilon_{3}(i \zeta)}{\varepsilon_{1}(i \zeta)+\varepsilon_{3}(i \zeta)}\right]\left[\frac{\varepsilon_{2}(i \zeta)-\varepsilon_{3}(i \zeta)}{\varepsilon_{2}(i \zeta)+\varepsilon_{3}(i \zeta)}\right] d \zeta
$$

Integrating all the values of the combined Hamaker coefficient, $\mathrm{A}_{132}$ gives an absolute value for the coefficient denoted by $A_{132 a b s}$ and applying Lifshitz derivation for van der Waals forces as in Equation 28.

The absolute value for the Hamaker coefficient could be derived by obtaining the mean of all the $A_{132}$ values got from the Lifshitz relation: ${ }^{21}$

$$
A_{132 a b s}=\frac{\sum_{i=1}^{N}\left(A_{132} i\right)}{N}
$$

Also

$$
A_{131 a b s}=\frac{\sum_{i=1}^{N}\left(A_{131} i\right)}{N}
$$

And

$$
A_{232 a b s}=\frac{\sum_{i=1}^{N}\left(A_{232} i\right)}{N}
$$

\section{Results, analysis and discussion}

\begin{tabular}{|c|c|c|c|c|c|c|}
\hline \multirow{2}{*}{ Sample type } & \multirow{2}{*}{$\begin{array}{l}\text { Wavelength, } \\
\lambda(\mathrm{nm}) \text { peak } \\
\text { values }\end{array}$} & \multicolumn{5}{|c|}{ Absorbance, ā peak values } \\
\hline & & Mtb positive & Mtb negative & Mtb/HIV co-infected & $\begin{array}{l}\text { Mtb/HIV } \\
\text { uninfected }\end{array}$ & Mean values \\
\hline & & $0.2918-0.7877$ & - & - & - & $0.4588 \pm 0.1468$ \\
\hline & & - & $0.2657-1.250 \mid$ & - & - & $0.6244 \pm 0.3545$ \\
\hline \multicolumn{7}{|l|}{ Sputum } \\
\hline & & - & - & $0.0231-0.0498$ & - & $0.0379 \pm 0.0108$ \\
\hline & 290 & & & & & \\
\hline & & - & - & - & $0.2657-1.2501$ & $0.6244 \pm 0.3545$ \\
\hline & & $0.0206-0.0736$ & - & - & - & $0.0496 \pm 0.0116$ \\
\hline & 320 & & & & & \\
\hline & & - & $0.0478-0.1148$ & - & - & $0.0784 \pm 0.0206$ \\
\hline \multicolumn{7}{|l|}{ Macrophage } \\
\hline & & - & - & $0.0152-0.0637$ & - & $0.0456 \pm 0.0106$ \\
\hline & 290 & & & & & \\
\hline & & - & - & - & $0.0478-0.1148$ & $0.0784 \pm 0.0206$ \\
\hline
\end{tabular}

The absorbance values were obtained as a function of wavelength and summarized in Table 1. It could be seen that the peak absorbance values of the various sputum samples and components vary in magnitude revealing the notable effect of the bacteria on them. The comparison between the positive and negative samples of the macrophages is imperative to this research. This is because Mtb actually attacks the macrophages by attaching itself to the macrophage cells.

Table I Comparison between peak absorbance values of mtb positive, mtb/HIV positive and negative sputum components respectively 
Table 1 reveals also the degree of variation between similar infected and uninfected sputum components at a glance for a clearer understanding. Equation 11 was used to obtain for each interacting system; $A_{i j}\left(A_{11}, A_{22}, A_{33}, A_{12}, A_{13}, A_{23}\right)$ by approximate change of variables using MatLab computation tools. This involved the numerical integration of Equation 11 for each wavelength from $230 \mathrm{~nm}$ to $950 \mathrm{~nm}$ for all the twenty samples in each category.
Analysis based on the particle-particle interaction mechanisms of the mathematical formulations outlined yielded values for the needed variables (such as the transmittance $T$, reflectance $R$, refractive index (real and imaginary) $n$, extinction coefficient $k$, absorption coefficient $\alpha$, dielectric constants (real and imaginary) $\varepsilon_{i j}$, Hamaker Constants $A_{i j}$, and Hamaker Coefficients $A_{132}$ were calculated and presented in Figure 3-5.

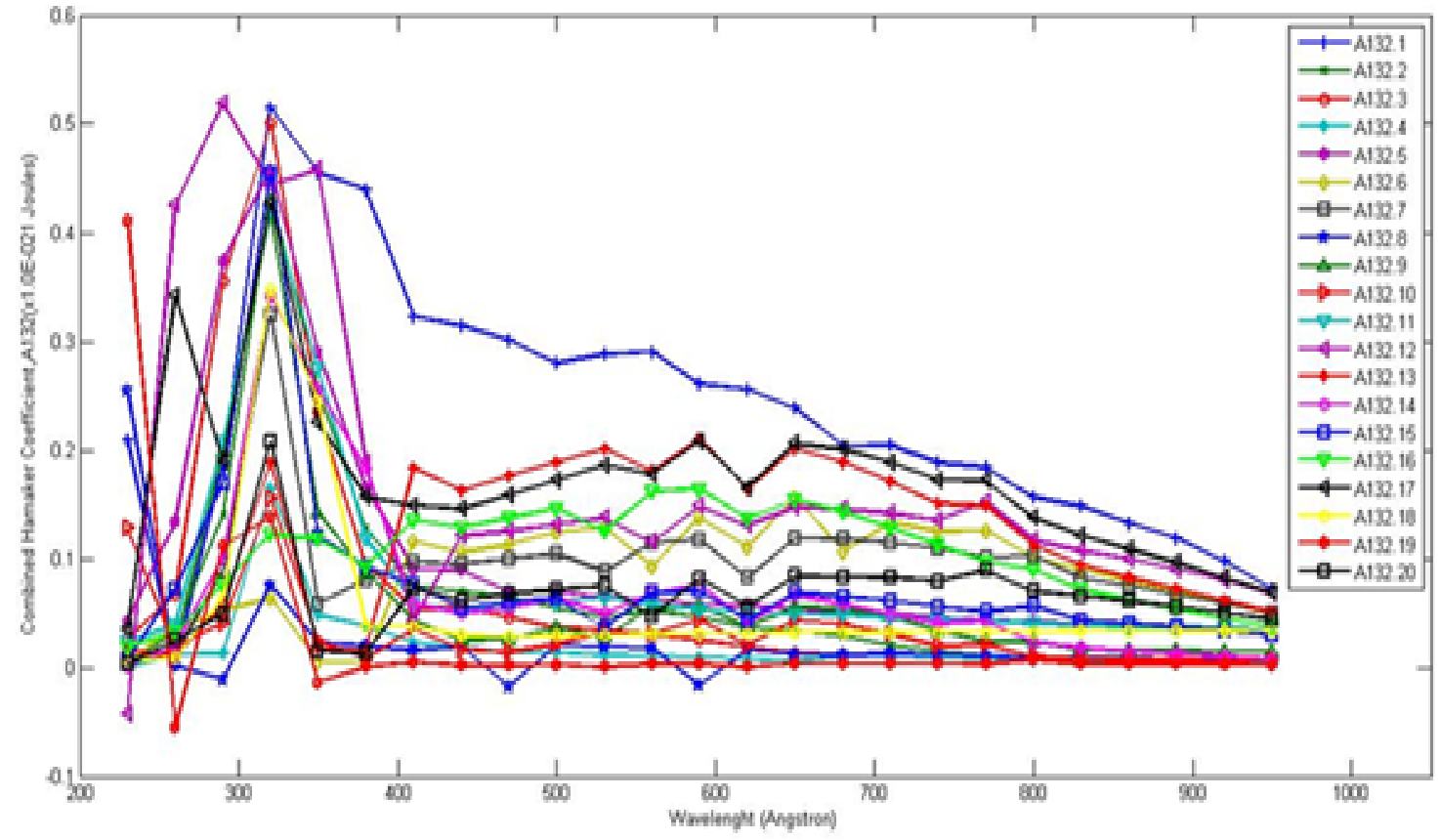

Figure 3 Variation of combined hamaker coefficient, $A_{132}$ with wavelength, $\lambda(n m)$ for the $m$ tb infected sputum samples.

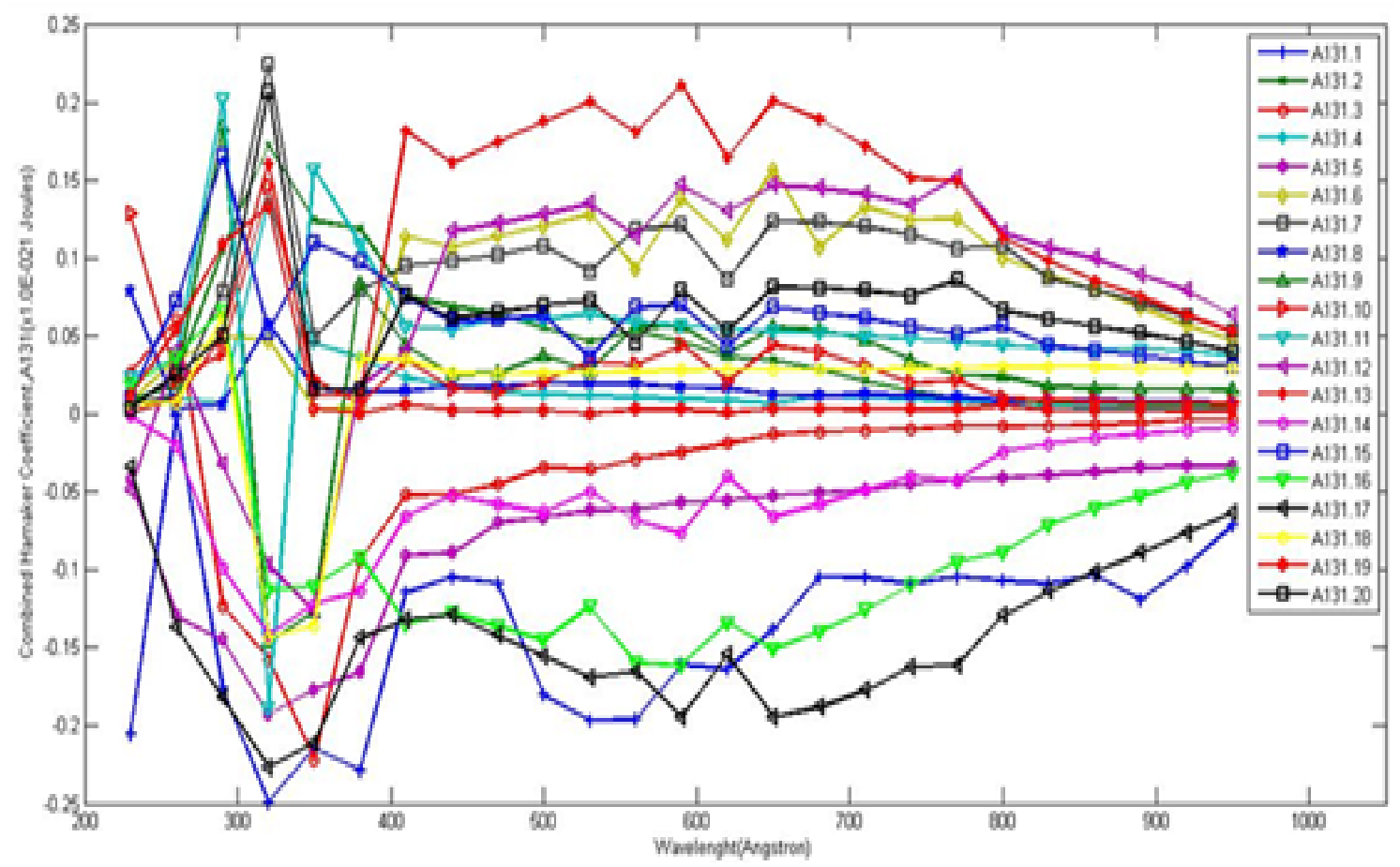

Figure 4 Variation of combined hamaker coefficient, $\mathrm{A}_{131}$ with wavelength, $\lambda(\mathrm{nm})$ for the $m$ tb uninfected sputum samples. 


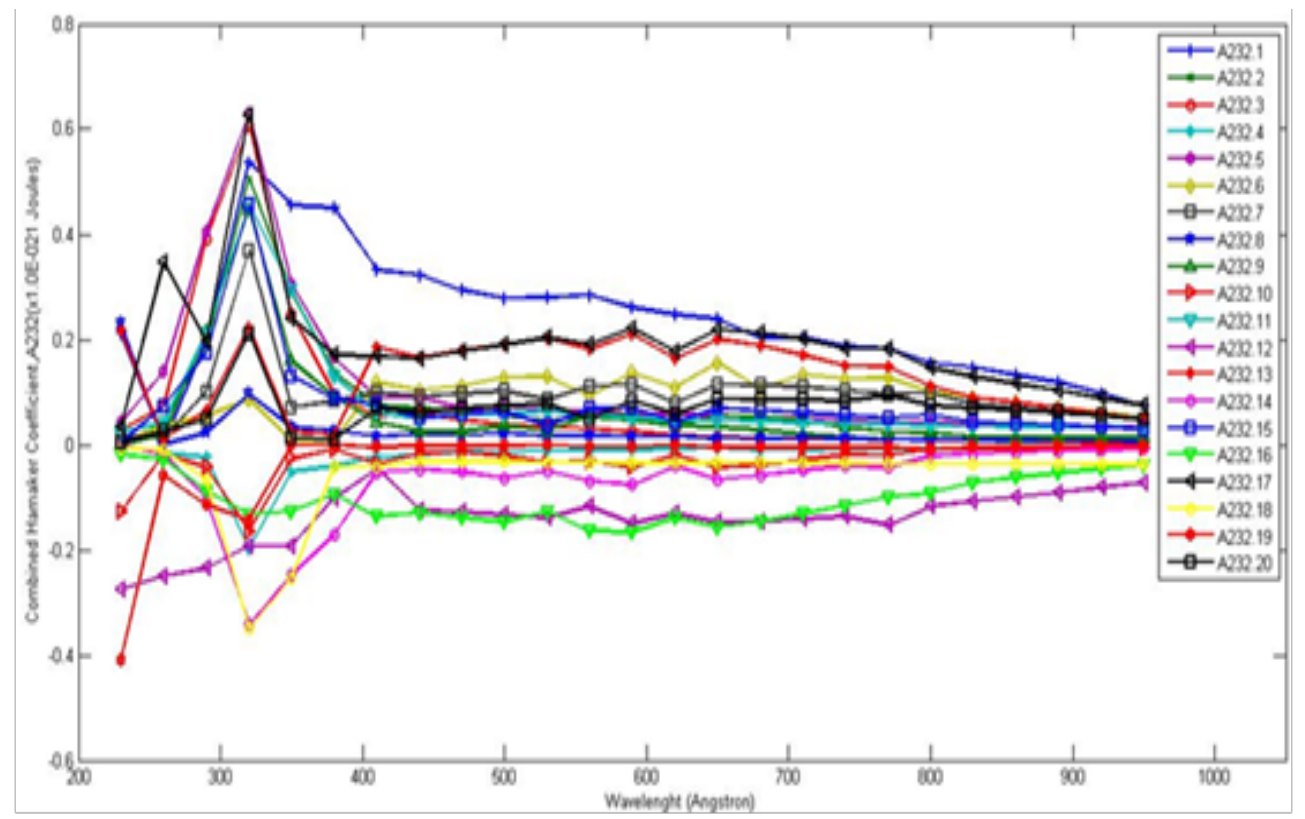

Figure 5 Variation of combined hamaker coefficient, $A_{232}$ with wavelength, $\lambda(\mathrm{nm})$ for the $m$ tb infected sputum samples.

The difference between the peak absorbance values of $m t b$ positive, $m t b / \mathrm{HIV}$ co-infected and negative sputum components respectively is an indication of how the bacteria affects the properties of the macrophage cells. The trend is such that the mean absorbance peak values of $m t b$ negative sputum samples are reduced by infection from $0.6244 \pm 0.3545$ to $0.4588 \pm 0.1468$ by a factor of about $26.5 \%$. In $m t b$ macrophage samples, the reduction is from $0.0784 \pm 0.0206$ to $0.0496 \pm 0.0116$, a factor of about $36.7 \%$. While in $m t b / \mathrm{HIV}$ coinfected macrophage samples, the reduction is from $0.0784 \pm 0.0206$ to $0.0456 \pm 0.0106$ by a factor of about $41.8 \%$. Comparing the mean absorbance peak values of $m t b$ positive sputum samples and the mean absorbance peak values of $m t b / H I V$ co-infected sputum samples; the results of the mean absorbance peak values reveal that the mean absorbance peak value of the $m t b / \mathrm{HIV}$ co-infected samples is generally reduced as compared to that of the mean absorbance peak values of the $m t b$ positive sputum samples (Table 1).

The macrophages are of particular interest to this research since the bacteria attacks this T-cells component which serves as receptor cells. Comparing the mean absorbance peak values of $m t b$ positive macrophage samples and the mean absorbance peak values of $m t b /$ HIV co-infected macrophage samples; the results of the mean absorbance peak values reveal that the mean absorbance peak values of the $m t b / \mathrm{HIV}$ co-infected samples is generally reduced by infection from $0.0784 \pm 0.0206$ to $0.0456 \pm 0.0106$ by a factor of about $41.8 \%$ as compared to that of the mean absorbance peak values of the $m t b$ positive macrophage sample with a factor of about $36.7 \%$. It could be seen that the reduction between the peak values absorbance (mean) of the sputum component is such that the macrophages reduced from $m t b$ to $m t b / \mathrm{HIV}$ by a factor of about $8.1 \%$. The reduction in the absorbance of the $m t b / H I V$ infected sputum samples reveals the role of the bacteria in drastically affecting the surface properties of the infected macrophage cells and specimens.

Figure 3-5 reveal the pattern of the combined Hamaker coefficients for the infected sputum samples with clear maxima and minima values occurring at various wavelengths. Harmonized values of the combined Hamaker coefficient became necessary to streamline the findings. This involves the integration of all the Hamaker coefficients first over each wavelength, secondly with each sputum sample in view to give the harmonized combined Hamaker coefficients, $\mathrm{A}_{132 \mathrm{har}}$.

Ultimately a single value of the Hamaker coefficient is essential in determining the final outcome. This is known as the absolute combined Hamaker coefficient, $A_{132 a b s}$ (Figure 3), $A_{13 \text { labs }}$ (Figure 4) and $\mathrm{A}_{232 \mathrm{abs}}$ (Figure 5). It is obtained by integrating all the values of the Hamaker coefficient to give a single value. Not much meaning could be made out of Figure 3-5. To generate more meaningful plot, average value for the given Hamaker coefficients of a given wavelength was determined from the data for the twenty samples. These results are given in Figure 6.

Figure 6 reveals the pattern of the average combined Hamaker coefficients, $A_{132}, A_{131}$ and $A_{232}$ for the sputum samples with clear peak values occurring at various wavelengths. The peak average values of $A_{132}$ and $A_{232}$ occur at wavelength of $320 \mathrm{~nm}$ with values of $0.30968 \times 10^{-}$ ${ }^{21} \mathrm{~J}$ and $0.18609 \times 10^{-21} \mathrm{~J}$ respectively, while the peak average value of $A_{131}$ occurs at wavelength of $290 \mathrm{~nm}$ with value of $0.03074 \times 10^{-21} \mathrm{~J}$. Energy level increases in average combined Hamaker coefficients, $\mathrm{A}_{132}$ and $\mathrm{A}_{232}$ which is the infected Hamaker coefficients as against the decreased energy level of the uninfected average combined Hamaker coefficient, $\mathrm{A}_{131}$. This is quite a significant phenomenon which explains away the fact of the effect of co-infection.

Table 2 reveal that the surface energy of the macrophages as computed in terms of Hamaker coefficients is less than the surface energy of the $M t b, M t b / \mathrm{HIV}$. This result also shows that the surface energy of the Mtb/HIV macrophage is less than that of $M t b$ macrophage. HIV has the tendency to reduce the energy with the consequence of increased viral loads and decreased immune systems. $t b$ is an opportunistic disease and in presence of HIV, the consequence is dreadful. Hence for $\mathrm{A}_{132}>0, \mathrm{~A}_{131 \text { (macropage) }}<\mathrm{A}_{232(\mathrm{M}-\mathrm{TB} / \mathrm{HIV})}$.

The mean of all values of $A_{11}$ and $A_{22}$ could be obtained and substituted into Equation 24 in order to derive a value for $\mathrm{A}_{33}$ at which 
$A_{132}$ is equal to zero in agreement with the earlier stated reasons.

Rearranging Equation 24 and making $\mathrm{A}_{33}$ subject of the formula:

$$
A_{33}=\left[\frac{2 \sqrt{A_{11}} \sqrt{A_{22}}-A_{132}}{\sqrt{A_{11}}+\sqrt{A_{22}}}\right]^{2}
$$

The mean of all the values of $\mathrm{A}_{11}$ and $\mathrm{A}_{22}$ respectively gave the absolute values of the Hamaker constants as: $\mathrm{A}_{11}=0.94188 \times 10^{-21} \mathrm{Joule}$ and $\mathrm{A}_{22}=0.96068 \times 10^{-21}$ Joule (for $m t b$ ); $\tilde{\mathrm{A}}_{11}=0.94188 \times 10^{-21}$ Joule and $\tilde{\mathrm{A}}_{22}=0.97862 \times 10^{-21}$ Joule (for $m t b / \mathrm{HIV}$ co-infection). Thus, inserting these values into Equation 29 and rendering $A_{132} \leq 0$ will give the critical value of $\mathrm{A}_{33 \mathrm{C}}$ that satisfies the condition for the combined Hamaker coefficient to be equal to or less than zero. Hence any value of $\mathrm{A}_{33}$ greater than the critical would be the desired value necessary to attain a negative combined Hamaker coefficient.

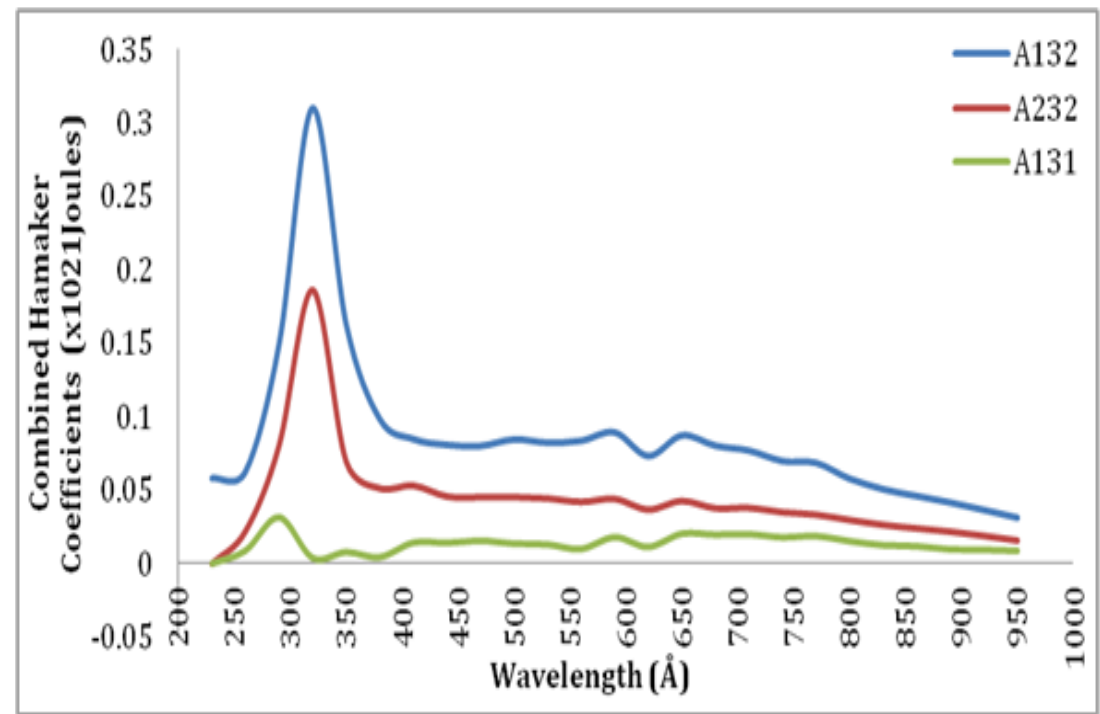

Figure 6 Variation of average combined hamaker coefficients, $A_{132} A_{131}$ and $A_{232}$ with wavelength, $\lambda(n m)$ for the $m$ tb infected sputum samples.

Table 2 Absolute values of Hamaker coefficients for uninfected and infected sputum

\begin{tabular}{|c|c|c|c|}
\hline \multicolumn{2}{|c|}{$\begin{array}{l}\text { Absolute values of } A_{131} \text { for uninfected } m t b \text { sputum and } A_{132} \text {, } \\
A_{232} \text { for infected } m \text { tb sputum }\end{array}$} & \multicolumn{2}{|c|}{$\begin{array}{l}\text { Absolute values of } \tilde{\mathbf{A}}_{131} \text { for uninfected } m \text { tb/HIV sputum and } \tilde{\mathbf{A}}_{132} \text {, } \\
\tilde{\mathbf{A}}_{232} \text { for infected } m \text { tb/HIV sputum }\end{array}$} \\
\hline Variable $\left(\times 10^{-21}\right.$ Joule) & Absolute value & Variable $\left(\times \mid 0^{-21} \mathrm{~J} J o u l e\right)$ & Absolute value \\
\hline$A_{131}$ & 0.10165 & $\tilde{A}_{131}$ & 0.10165 \\
\hline$A_{232}$ & 0.24986 & $\tilde{A}_{232}$ & 0.20474 \\
\hline$A_{132}$ & 0.21631 & $\tilde{A}_{132}$ & 0.18825 \\
\hline
\end{tabular}

Thus, the critical absolute Hamaker constant $A_{33 \mathrm{C}}$ for the sputum which renders the $A_{132}$ negative is given as: $A_{33 C}=0.9527 \times 10^{-21}$ Joule (for $m t b$ ) and $\tilde{\mathrm{A}}_{33 \mathrm{C}}=0.9598 \times 10^{-21}$ Joule (for $m t b / \mathrm{HIV}$ co-infection). Thus for negative combined Hamaker coefficient $A_{132}, \tilde{A}_{132}$ of the infected $m t b, m t b / \mathrm{HIV}$ sputum to be attained respectively, the combined Hamaker constant of the sputum as the intervening medium $\mathrm{A}_{33}, \tilde{\mathrm{A}}_{33}$ respectively should be of the magnitude: $\mathrm{A}_{33 \mathrm{C}} \geq 0.9527 \times 10^{-21}$ Joule and $\tilde{\mathrm{A}}_{33 \mathrm{C}} \geq 0.9598 \times 10^{-21}$ Joule respectively. Inserting the above values of $\mathrm{A}_{33}, \tilde{\mathrm{A}}_{33 \mathrm{C}}$ into Equation 24 would yield negative values for $\mathrm{A}_{132}, \tilde{\mathrm{A}}_{132}^{33}$ respectively as follows: $\mathrm{A}_{132}=-0.22669 \mathrm{x} 10^{-25}$ Joule (when $\mathrm{A}_{33}=0.9527 \times 10^{-21}$ Joule) and $\tilde{\mathrm{A}}_{132}=-0.08786 \times 10^{-25}$ Joule (when $\mathrm{A}_{33}=0.9598 \times 10^{-21} \mathrm{Joule}$ ). A mathematical expression for harmonized Hamaker coefficients was given as follows:22

$$
\varphi_{t}=\int_{0}^{\infty}\left(A_{132 \max }-A_{132 h a r}\right) d \lambda
$$

Where $\Phi_{t}=$ the integral of the difference between $\mathrm{A}_{132 \max }$ and $\mathrm{A}_{132 \mathrm{hars}}$.

Thus, from the deductions made from this work the following result was got;

$$
A_{132 \text { har }} \approx 0.4725\left(A_{132 \max }\right)
$$

Hence

$$
A_{132 \max } \approx \frac{1}{0.4725}\left(A_{132 \text { har }}\right)
$$

$$
A_{132 \text { har }}=\varphi_{t} A_{132 \max }
$$

Where

It could easily be seen that in finding a solution for the absolute Hamaker coefficient, the maxima and harmonized values of Hamaker 
coefficient could be used. This approximate method thus serves as an alternative method for obtaining a reliable value for the absolute combined Hamaker coefficient for human sputum systems and could well be relevant to other biological and particulate systems. This latter statement needs verification and could be taken up as a new research area in this field. The single value of the Hamaker coefficient is necessary in determining the final conclusion. Harmonized values of the combined Hamaker coefficient is required to rationalize the findings. This involves the integration of all the Hamaker coefficients over each wavelength, with each sputum sample in view to give the harmonized combined Hamaker coefficients, $\mathrm{A}_{132 \mathrm{har}}$ for both $m t b$ and $m t b / \mathrm{HIV}$ co-infection. The harmonized values of the combined Hamaker coefficient for each infected and uninfected sputum samples are shown in Table 3 alongside with that of the absolute combined Hamaker coefficient for both $m t b$ and $m t b / \mathrm{HIV}$ co-infection. The interaction systems of $m t b$ with the HIV sputum samples gave a positive absolute combined Hamaker coefficient, $\mathrm{A}_{132}$ which entails attractive van der Waals forces.

The positive sense of the absolute combined Hamaker coefficient obtained is in agreement with the statement of Achebe et al. ${ }^{22}$ However, the positive values of the absolute combined Hamaker coefficient $\mathrm{A}_{131}$ for $m t b$ interacting with HIV negative sputum samples as shown in Table 3 indicate that there is an attraction between the interacting bodies. This is so because the positive sense of the absolute combined Hamaker coefficient shows that the van der Waals forces of the interacting systems are attractive. A comparison in Table 3 of the maxima $\mathrm{A}_{132 \max }$ and the harmonized $\mathrm{A}_{132 \mathrm{har}}$ values of the combined Hamaker coefficient shows a direct association between the two. Difference in terms of the magnitude only was recorded. However, this research work is limited to the role the van der Waals forces play in the process and progress of $m t b / \mathrm{HIV}$ co-infection, the mechanism involved and the contribution its study could offer in the chase for a feasible solution to the overwhelming condition.

Table 3 Comparison of the values of the absolute combined Hamaker coefficients, and the harmonized combined Hamaker coefficients, of the Mtb, Mtb/HIV on both infected and uninfected sputum

\begin{tabular}{|c|c|c|c|}
\hline \multicolumn{2}{|c|}{ Infected sputum (mtb) } & \multicolumn{2}{|c|}{ Uninfected sputum (mtb) } \\
\hline$A_{132 a b s}\left(\times 10^{-21}\right.$ Joule $)$ & $A_{132 \mathrm{har}}\left(\times 10^{-21}\right.$ Joule $)$ & $A_{13 \text { labs }}\left(\times 10^{-21}\right.$ Joule $)$ & $A_{13 \mid \text { har }}\left(\times \mid 0^{-21}\right.$ Joule $)$ \\
\hline 0.21631 & 0.24509 & 0.10165 & 0.10589 \\
\hline \multicolumn{2}{|c|}{ infected sputum (mtb/HIV co-infection) } & \multicolumn{2}{|c|}{ uninfected sputum (mtb/HIV Co-Infection) } \\
\hline$\tilde{A}_{132 a b s}\left(\times 10^{-21}\right.$ Joule $)$ & $\tilde{A}_{132 \text { har }}\left(\times 10^{-21}\right.$ Joule $)$ & $\tilde{A}_{13 \text { labs }}\left(\times 10^{-21}\right.$ Joule $)$ & $\tilde{\mathrm{A}}_{13 \text { lhar }}\left(\times 10^{-21} \mathrm{Joule}\right)$ \\
\hline 0.18825 & 0.201 & 0.09627 & 0.10589 \\
\hline
\end{tabular}

\section{Conclusion}

\section{Acknowledgements}

None.

\section{Conflict of interest}

The author declares no conflict of interest.

\section{References}

1. Bauer AL, Hogue IB, Marino S, et al. The effects of HIV-1 infection on latent tuberculosis. Math Model Nat Phenom. 2008;3(7):229-266.

2. Paul Nunn, Brian Williams, Katherine Floyd, et al. Tuberculosis control in the era of HIV. Nature Reviews Immunology. 2005;5(10):819-826.

3. World health organization. Global tuberculosis control. Geneva, Switzerland: WHO report; 2010.

4. Aandahl RZ, Reyes JF, Sisson SA, et al. A model-based bayesian estimation of the rate of evolution of vntr loci in mycobacterium tuberculosis. PLoS Comput Biol. 2012;8(6):e1002573.

5. Chukwuneke JL, Chukwuneke PC, Okolie PC, et al. Hamaker coefficient concept: the application to the mycobacterium tuberculosis-macrophage interactions mechanism. Advances in Bioscience and Bioengineering. 2016;4(1):1-20.

6. Gyasi Agyei KA, Agyei AG, Obeng Denteh W. Mathematical modelling of the epidemiology of tuberculosis in the ashanti region of Ghana. British Journal of Mathematics \& Computer Science. 2014;4(3):375-393.

7. World health organization. Global tuberculosis. Geneva, Switzerland: WHO report; 2012. the condition of $\mathrm{A}_{33}$ above, possible additive(s) to the system (in form of drugs) to the sputum as intervening medium should be required. absolute combined Hamaker coefficient $\mathrm{A}_{132 a b s}$ negative. A model for the infection mechanism was built-up employing the principle of particle interaction. Mathematically, it was derived as $\mathrm{A}_{33} \geq 0.9527 \times 10$ ${ }^{21}$ Joule which satisfies this condition for negative $A_{132 a b s}$. To achieve 
8. World health organization. Tuberculosis. Geneva, Switzerland: WHO information: Fact Sheets; 2005.

9. Tsai MC, Chakravarty S, Zhu G, et al. Characterization of the tuberculous granuloma in murine and human lungs: cellular composition and relative tissue oxygen tension. Cell Microbiol. 2006;8(2):218-232.

10. Van Oss CJ, Visser J, Absolom DR, et al. The concept of negative hamaker coefficients, II. Thermodynamics, experimental evidence and applications. Advances in Colloid and Interface Science. 1983;18(34):133-148.

11. Stuart AE. Phagocytic engulfment and cell adhesiveness as surface phenomena. J Clin Pathol. 1977;30(6):592.

12. Absolom DR, Francis DW, Zingg W, et al. Phagocytosis of bacteria by platelets: surface thermodynamics. Journal of Colloid and Interface Science. 1982;85(1):168-177.

13. Omenyi SN. Attraction and Repulsion of particles by Solidification Fronts. PhD Dissertation, Canada: University of Toronto; 1978. p. 33-34.

14. Visser J. The concept of negative hamaker coefficients. 1. History and present status. Advances in Colloid and Interface Science. 1981;15(2):157-169.
15. Achebe CH, Sinebe JE, Chukwuneke JL, et al. Surface energetics of mycobacterium tuberculosis -macrophage interactions. Journal of Biomedical Engineering and Medical Imaging. 2015;2(6):49-61.

16. Hamaker HC. The London-van der Waals attraction between spherical particles. Physica. 1937;4(10):1058-1072.

17. Lifshitz, EM. The theory of molecular attractive forces between solids. Soviet Phys. JETP. 1956;2(1):73.

18. David B Hough, Lee R White. The calculation of Hamaker constants from Liftshitz theory with applications to wetting phenomena. Advances in Colloid and Interface Science. 1980;14(1):3-41.

19. Robinson TS. Optical constants by reflection. Proceedings of the physical society. Section. 1952;B 65(11):B910.

20. Kittel Charles. Introduction to Solid State Physics. 7th ed. New York, USA: John Willey and sons Inc; 1996. 308 p.

21. Achebe CH. Human Immunodeficiency Virus (HIV)-Blood Interactions: Surface Thermodynamics Approach. Nigeria: Nnamdi azikiwe university; 2010. p. 1-120.

22. Achebe $\mathrm{CH}$, Omenyi SN, Manafa OP, et al. Human Immunodeficiency Virus (HIV)-Blood Interactions: Surface Thermodynamics Approach. Proceedings of the international multi-conference of engineers and computer scientists, Hong Kong: IMECS; 2012. p. 136-141. 